\title{
Prevalence of TMJ Disorders in Competitive Swimmers: A Cross Sectional Study
}

\author{
Ajay Kumar ${ }^{1}$, Arya Dharan B ${ }^{* 2}$, Deepak Kumar Pradhan ${ }^{3}$ \\ ${ }^{1}$ Professor, Department of Physiotherapy, Srinivasa University, Mangalore, Karnataka, India \\ ${ }^{2}$ Post-Graduate Student, Department of Physiotherapy, Srinivasa University, Mangalore, Karnataka, India \\ ${ }^{3}$ Assistant Professor, Department of Physiotherapy, Srinivasa University, Mangalore, Karnataka, India
}

\begin{tabular}{|c|c|}
\hline DOI: $10.36348 / J A S P E .2019 . v 02 \mathrm{i} 07.003$ & \\
\hline
\end{tabular}

*Corresponding author: Arya Dharan B

Abstract

Swimming is an unique sport. It is the combination of upper limb and lower extremity strength exercise and also cardiovascular training in non-weight bearing environment ' In competitive swimmers, there are four strokes freestyle, butterfly, backstroke, and breaststroke. Competitive swimmers trains 10,000 to 20,000 meter per day. At an average of 8 to $10 \mathrm{arm}$ cycles per 25 meter. Swimmers push their body to the limits of the functions so normal variations in anatomy or biomechanics and poor technique may lead to overuse injuries and micro trauma. The most common swimming injuries are shoulder, neck and back. The prevalence of musculoskeletal injuries in competitive swimmers is, shoulder $37 \%$, knee $28 \%$, spine $22 \%$ and foot and ankle is $19 \%$. Limited number of studies are available to find out the prevalence of TMJ disorders in competitive swimmers. This study puts in an effort to find the same.

Keywords: TMJ, TMD, Competitive, Swimmers.

Copyright @ 2019: This is an open-access article distributed under the terms of the Creative Commons Attribution license which permits unrestricted use, distribution, and reproduction in any medium for non-commercial use (NonCommercial, or CC-BY-NC) provided the original author and source are credited.

\section{INTRODUCTION}

Temporomandibular joint (TMJ) is complex junction. It consists of three articular surface mandibular fossa, articular tubercle and head of the mandible [1-3]. The main function of the joint is opening and closing of the mouth [8]. The important muscles are involved in TMJ movements are the digastricus, masseter, lateral pterygoid and masticator muscles such as supra hyoid and infra hyoid. In temporomandibular joint disorder (TMD), the TMD, masticatory muscles or both are involved. It can cause non dental pain in orofacial region. Impaired joint mobility clicking or crepitus, pain in the TMJ and ear, Eustachian tube dysfunction and dizzy spells. The predisposing factors are joint laxity, anatomical variations, capsular or muscular inflammation, repetitive motion and static articular stress [4, 5].

TMJ dysfunction is multifactorial disorder commonly associated with masticatory and articular disabilities and it is the combination of physiological structural and postural factors leads to derangement of functional balance between structures of TMJ. The association of pain is an important symptom has leads to need for investigation of epidemiology. However, swimming is a unique sport require combination action of both the extremities and trunk motions. According to previous studies there is a high demand on neck rotation and upper limb activities for the propulsion of the body inside the water and which negative synchronized activities of jaw and mastication structure. The importance of epidemiology of mastication and its knowledge requires effective diagnosis and therapeutic planning to completely all alleviate signs and symptoms. Previous studies has evaluated the prevalence of TMJ disorder among various population But till date there is no studies have been attempted to find out the prevalence of TMD among competitive swimmers through there is a greater demand on TMJ structure due to their swimming mechanics. So there is a need.

AIM OF THE STUDY

To find out the prevalence of TMD in competitive swimmers..

\section{MATERIALS AND METHODOLOGY \\ Study Design: A cross sectional study}

Study Population: competitive swimmers

Study Sample Size: 300 
Study Setting: various swimming clubs in South India.

Study duration: April 2017- May 2019

\section{CRITERIA FOR SELECTION [5-7] \\ Inclusion Criteria}

- Competitive swimmers aged between 15-35 year.

- Regularly train at least 5 times per week,

- Distance 6-8 miles per practice.

- Subjects with TMD, 6 month history of pain.

\section{Exclusion Criteria}

- History of spinal surgery

- Any previous incidence of TMD or dental issues (before coming to swimming)

- Previous history of major trauma (motor vehicle accidents)

\section{Procedure}

Various swimming clubs in South India will be contacted and approached. This will be followed by distribution and selection of swimmers using screening form. If the swimmers met with the selection criteria they will be included our study followed by informed consent will be obtained prior to voluntary participation in the study. If the swimmers present with any TMJ pain or discomfort it will be further assessed according to the RDC/TMD criteria to confirm the diagnosis. Then the data will be used for further analysis.

\section{To Measure Temporomandibular Disorders}

TMD will be assessed by Research diagnostic criteria for temporomandibular disorders (RDC/TMD). According to the RDC/TMD qualified therapist will be assess the subject (based on the previous studies) [8].

\section{Data Analysis}

The data will be collected and all the variables and its characteristics will be described using tables and graphs. All data will be coded and entered in to the software SPSS version 16.0 (statistical package for social sciences) in windows. Descriptive analysis will be done by finding mean and standard deviation of all the samples. The Chi-square test will be used for the analysis of data.

\section{OBSERVATION AND RESULTS}

A total of 300 swimmers $(60 \%$ were male and $40 \%$ were females were enrolled in this a prospective, cross sectional study).

Prevalence of the symptoms of TMD was calculated:

The prevalence of TMD was observed in $41 \%$ $(\mathrm{n}=123)$ (71 males and 52 females).

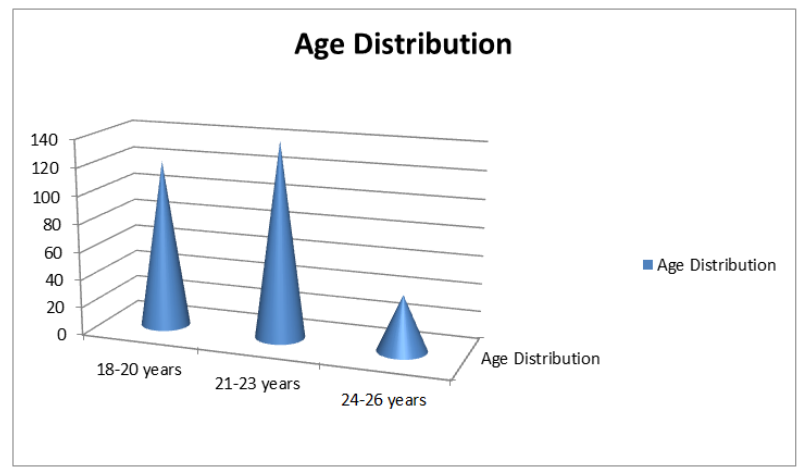

Fig-1: Age Distribution

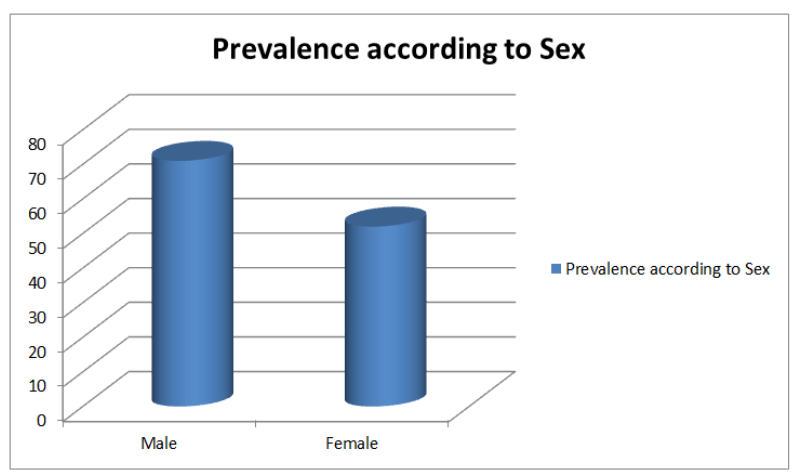

Fig-2: Male and Female Prevalence

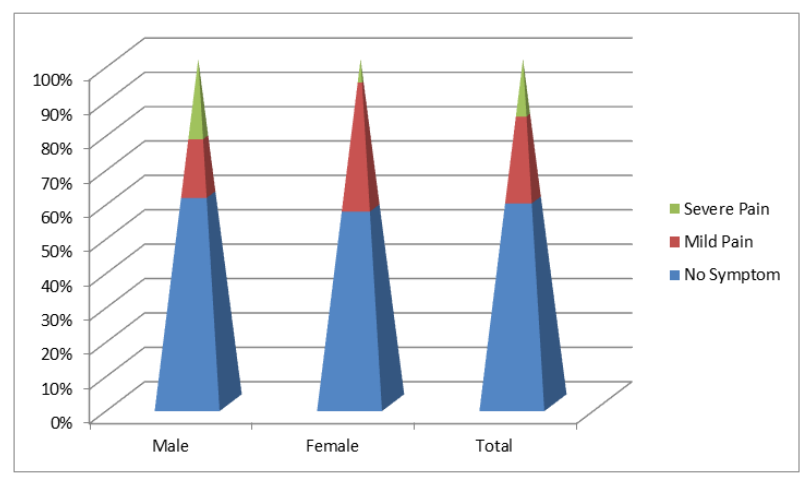

Fig-3: Severity

\section{DISCUSSION}

TMD has commonly been associated with other conditions affecting the head and neck region. Such as headache, neck pain and neck muscular dysfunction. According to the previous studies there is a strong relationship exists between neck disability and jaw disability $[9,5,6]$. Temporomandibular joint disorders presented with abnormal pattern of contraction of cervical flexor muscles and an increased fatigability of the flexors and extensors cervical muscles. And also found that cervical mobility and functional exercise and head and neck postural correction decreases the symptoms in patients with TMD $[9,7,10]$.

The supra hyoid and infra hyoid affect the balance between the flexors and extensors of the head and neck dysfunction in either these muscles or cervical muscles can easily disturb in this normal balance [4]. 
Electromyography studies are saying increased level of cervical backward bending and cervical flexion. The backward bending increases the activity of temporalis muscle and cervical flexion increases the activity of the masseter and digastric. Excessive mandibular shuttling between opening and closing for functional activities such as eating, leads to joint hypermobility because TMJ capsule is stretched. Increases muscular activity in the anterior cervical (longus coli) and hyoid muscle will turn in cause tightness in the through and difficulty in swallowing. Mouth breathing is an important contributing factor. Breathing through the mouth facilitate forward head posture and a low and forward tongue position $[4,11]$.

During swimming nasal breathing is left and mostly favor of an oro-nasal respiration. That leads to less or more mandibular movements with variation of supra and infra hyoid muscle, and of cervico-brachial muscle and their variation can leads to alternative of position of teeth and bone bases and may develop dental mal occlusion, dysfunction of temporomandibular joint intra or extra capsular type $[10,11]$.

Other predisposing factors that may cause TMD is variety of neurological and muscular disorder, bone diseases, tumors, infections, psychogenic disorder, disease causing disturbance of the occlusion of the teeth or supporting structures, faulty habits of the jaw, and orofacial imbalance $[9,11]$.

\section{CONCLUSION}

The prevalence of the TMD is high in professional swimmers. It needs a case control or cohort study for further proofs so as to take necessary steps to decrease the detrimental effects.

\section{REFERENCE}

1. Pollard, H., \& Fernandez, M. (2004). Spinal musculoskeletal injuries associated with swimming: a discussion of technique. Australasian Chiropractic \& Osteopathy, 12(2), 72-80.

2. Wanivenhaus, F., Fox, A. J., Chaudhury, S., \& Rodeo, S. A. (2012). Epidemiology of injuries and prevention strategies in competitive swimmers. Sports health, 4(3), 246-251.

3. Johnson, J. N., Gauvin, J., \& Fredericson, M. (2003). Swimming biomechanics and injury prevention: new stroke techniques and medical considerations. The Physician and sportsmedicine, 31(1), 41-46.

4. Levangie, P. K., \& Norkin, C. C. (2016). Joint struture and function A Comprehensive analysis. 4th edition. New Delhi: Jaypee Brother Medical Publishers (P) Ltd.

5. Olivo, S. A., Fuentes, J., Major, P. W., Warren, S., Thie, N. M. R., \& Magee, D. J. (2010). The association between neck disability and jaw disability. Journal of oral rehabilitation, 37(9), 670-679.

6. Rocha, C. P., Croci, C. S., \& Caria, P. H. F. (2013). Is there relationship between temporomandibular disorders and head and cervical posture? A systematic review. Journal of oral rehabilitation, 40(11), 875-881.

7. Walczyńska-Dragon, K., Baron, S., NiteckaBuchta, A., \& Tkacz, E. (2014). Correlation between TMD and cervical spine pain and mobility: is the whole body balance TMJ related?. BioMed research international, 2014:1-7.

8. Schiffman, E., Ohrbach, R., Truelove, E., Look, J., Anderson, G., Goulet, J. P., ... \& Svensson, P. (2014). Diagnostic criteria for temporomandibular disorders (DC/TMD) for clinical and research applications: recommendations of the International RDC/TMD Consortium Network and Orofacial Pain Special Interest Group. Journal of oral \& facial pain and headache, 28(1), 6-27.

9. Yuill, E., \& Howitt, S. D. (2009). Temporomandibular joint: conservative care of TMJ dysfunction in a competitive swimmer. The Journal of the Canadian Chiropractic Association, 53(3), 165-172.

10. Del Grosso, F., Nannelli, P., D’Ercole, S., Tieri, M., Martinelli, D., Di Nicola, M., \& Tripodi, D. (2015). Effects of Swimming on Stomatognathic System. Ann Sports Med Res, 2(9), 1052.

11. Kessler, R. M., \& Hertling, D. (1996). Management of common musculoskeletal disorders physical therapy principels and methods. 6th edition. Philadelphia: Lippincott, J. B. (P) Ltd. 